\title{
Phasor measurement units, WAMS, and their applications in protection and control of power systems
}

\author{
Arun G. PHADKE ${ }^{1}$, Tianshu BI ${ }^{2}$
}

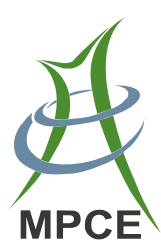

\begin{abstract}
The paper provides a short history of the phasor measurement unit (PMU) concept. The origin of PMU is traced to the work on developing computer based distance relay using symmetrical component theory. PMUs evolved from a portion of this relay architecture. The need for synchronization using global positioning system (GPS) is discussed, and the wide area measurement system (WAMS) utilizing PMU signals is described. A number of applications of this technology are discussed, and an account of WAMS activities in many countries around the world are provided.
\end{abstract}

Keywords Phasor measurement unit (PMU), Wide area measurement system (WAMS), Global positioning system (GPS), IEEE standards, Instrument transformers, Calibration, Parameter estimation

\section{Introduction}

Phasor measurement units (PMUs) evolved out of early work on computer based relaying performed at the American Electric Power (AEP) Corporation. The first author

CrossCheck date: 17 April 2018

Received: 12 February 2018/Accepted: 7 May 2018/Published online: 5 July 2018

(C) The Author(s) 2018

$\triangle$ Arun G. PHADKE

aphadke@vt.edu

Tianshu BI

tsbi@ncepu.edu.cn

1 Virginia Tech, Blacksburg, Virginia 24061, USA

2 North China Electric Power University, Beijing, China had joined AEP in 1969, and was soon given the assignment of developing a digital computer based distance relay for protecting overhead transmission lines. It was expected that the new relay will have performance comparable to that of best available analog relays. In particular, it was required that the new relay have a nominal operating time of one cycle.

In order to accomplish this task a team of engineers was assembled at AEP. One of the leading contributors to this task was Stanley H. Horowitz, the head of relaying department at AEP. Without his enthusiastic support and active participation in the project the relay development would not have progressed as rapidly as it did. In addition to Mr. Horowitz, the research team included Mohamed Ibrahim, Ted Hlibka, Mark Adamiak, Jerry Jauch and Mike Price. Several other graduate students from various universities joined the effort as co-operating students as part of their studies towards an engineering degree.

In mid-1970s we received at AEP Professor James Thorp of Cornell University as a sabbatical visitor. Ever since then, Professor Thorp has been a stalwart in computer relaying research and in PMU research. Even to this day he contributes to the research at Virginia Tech, where he became a Department Head of Electrical and Computer Engineering Department in about 2003.

One of the first tasks undertaken by our group was the construction of an analog network simulator with which the behavior of a complex electric power network could be simulated. The simulator is shown in Appendix A Fig. A1.

The simulator consisted of pi-section models of transmission lines, and various generator and transformer models. Complex three phase networks were modeled, and various switching operations representing faults and circuit breaker operation could be simulated. The simulator was 
mainly used to represent power system phenomena which affect the behavior of relays: time of fault inception, voltage and current transients, DC offset in currents etc. were represented with high precision.

In 1970s the computers available for implementing relaying algorithms were mini-computers. These were fairly large machines with large rotating discs and limited high-speed memory units. The research team selected IBM System7 for the development of the relay. Computer technology of that day had relatively slow instruction execution time, and it was soon discovered that in order to accommodate required computations for a three phase distance relay within one cycle of the $60 \mathrm{~Hz}$ system was difficult.

In order to overcome the problem of available computing time window the first author developed a new relaying principle based on using symmetrical component voltages and currents instead of the traditional phase quantities. This led to a principle known as symmetrical component distance relay (SCDR). The main point of this development was the use of a single complex equation to cover the protection of all ten possible faults on a three phase transmission line.

Figure 1 explains the principle of SCDR. Samples of current and voltage inputs are taken, and using appropriate Nyquist filters and discrete Fourier transform (DFT) phasors of voltages and currents are calculated. The $k$ in the figure stands for a single equation using symmetrical components which calculates distance to any fault on the line. A relay based on the principle of SCDR was implemented on the IBM System7 and was installed in the MattFunk substation of the Appalachian Power Company.

In time micro-computers became available. These were much smaller in size than the mini-computers, and their computing capabilities were much improved. One could go back to the traditional relaying algorithms using phase quantities rather than symmetrical components, and be able to fit the computation within one cycle of the power frequency.

However, it was soon realized that although the SCDR was no longer needed because of the time pressure, there was a great advantage in salvaging the portion of the SCDR

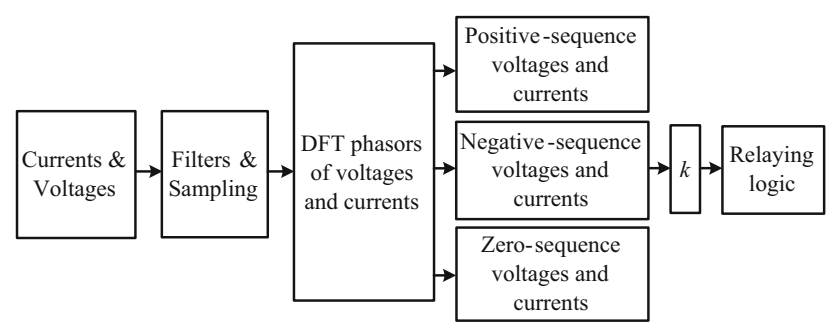

Fig. 1 Principle of SCDR which computed the sequence components of voltages and currents. The portion of SCDR illustrated in Fig. 1 up to the computation of positive-sequence voltages and currents was pulled out as a stand-alone measurement unit which could measure with great accuracy the positive-sequence voltages and currents in one period of the fundamental frequency. Furthermore, the measurement could be repeated every cycle, thus providing the user a continuous picture of these quantities making it possible for the first time the real-time observation of the dynamic phenomena taking place on the power system.

Around 1990 work was seriously begun to create a stand-alone PMU capable of measuring positive-sequence voltages and currents using global positioning system (GPS) satellite transmission to synchronize the measurements across the power system. This effort was led by several graduate students, among them Miroslav Begovic and Virgilio Centeno. Centeno was the principal designer of the PMU hardware and software, and with his help the first complete PMUs were built at Virginia Tech. First such units are shown in Fig. 2. After graduation, Centeno joined Macrodyne Corporation where with the help of its President R.J. Murphy PMUs began to be produced commercially.

\section{Phasor measurement techniques}

A pure sinusoid quantity given by $x(t)=X_{\mathrm{m}} \cos (\omega t+$ $\theta)$ and its phasor representation $\boldsymbol{X}=\left(X_{\mathrm{m}} / \sqrt{2}\right) \mathrm{e}^{\mathrm{j} \theta}=$ $\left(X_{\mathrm{m}} / \sqrt{2}\right)(\cos \theta+\mathrm{j} \sin \theta)$ are illustrated in Fig. 3. The aim of phasor estimation technique is just to acquire the phasor representation. Samples of waveform data are collected over a data window which is normally one period of the fundamental frequency of the power system. In early days a sampling rate of 12 times a cycle $(720 \mathrm{~Hz}$ for the $60 \mathrm{~Hz}$

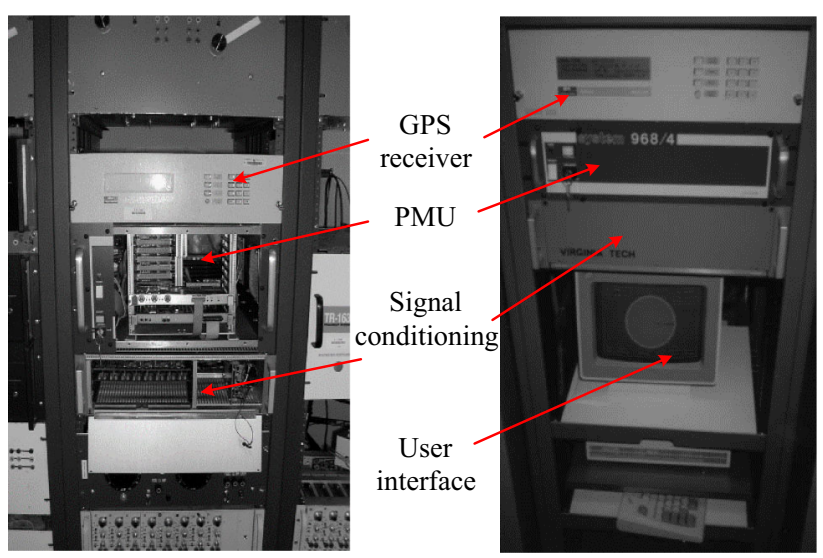

Fig. 2 First set of PMUs built at Virginia Tech 


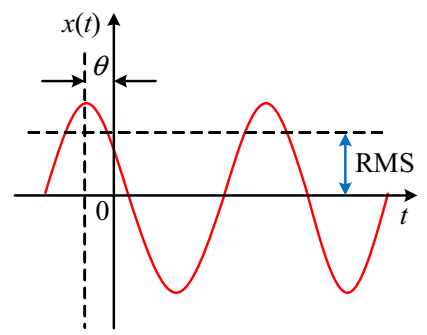

(a)

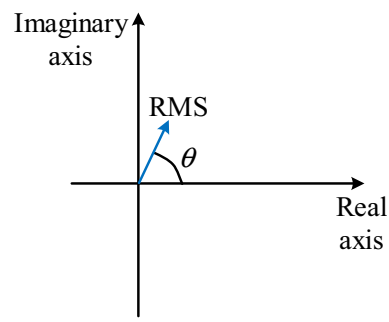

(b)
Fig. 3 A sinusoid and its representation as a phasor

system) was commonly used. Much higher sampling rates are currently used in commercial PMUs.

Taking samples over one period of the nominal power system frequency, the phasor is estimated by the familiar DFT:

$\bar{X}=X_{r}+\mathrm{j} X_{i}=\frac{\sqrt{2}}{N} \sum_{n=1}^{N}\left(x_{n} \cos \frac{2 n \pi}{N}+\mathrm{j} x_{n} \sin \frac{2 n \pi}{N}\right)$

where $X_{r}$ and $X_{i}$ are real and imaginary parts of the actual synchrophasor; $x_{n}$ is the data sample; and $N$ is the number of samples in one period. The phasor has the magnitude of the root mean square (RMS) value of the input signal, and the phase angle is the angle between the instant when the signal is measured and the peak of the input signal.

If the frequency of the input signal is different from the nominal frequency, a magnitude and angle correction must be applied to account for the frequency deviation. Also it is assumed that the input signal has been filtered according the requirement of the Nyquist criterion for the selected sampling rate.

Often the estimation data window needs to be different from one period, in which case a multiple cycle window may be used. Unlike in relaying applications, it is not considered necessary that a fractional cycle window be used for phasor estimation in PMU applications.

One of the early applications of PMU measurements was to estimate the frequency and rate of change of frequency (ROCOF). The early attempts used the difference between two consecutive phase angles divided by the time difference to estimate the frequency. As can be expected, this leads to a noisy estimate of the frequency, and the ROCOF is hopelessly noisy. A better approach is to measure several consecutive phase angles - for example over 3-6 periods, and make a polynomial fit to these angles. A second order polynomial is usually sufficient, and very good estimates of the frequency and ROCOF can be found by differentiating the polynomial.

\section{Synchronization}

Phasor is a complex representation of pure sinusoidal signal, which contains both amplitude $(A)$ and angle $(\theta)$. The phase angle depends upon the choice of the axis $t=0$, which indicates the timing reference. By synchronizing the sampling processes for different signals which may be hundreds of miles apart, it is possible to put their phasors on the same phasor diagram, achieving the purpose of comparison and analysis.

To achieve a common timing reference for the synchrophasor acquisition process, it is essential to have a source of accurate timing signals. In early 1980s, LORANC, Geostationary Operational Environmental Satellites (GOES) satellite transmissions, and the HBG radio transmissions in Europe [1-3] were used to obtain synchronization of reference time at different locations in a power system. The next available positive-going zero-crossing of a phase voltage was used to estimate the local phase angle with respect to the time reference. Using the difference of measured angles on a common reference at two locations, the phase angle difference between voltages at two buses was established.

With the development of GPS technology, it offers the most effective way of synchronizing power system measurements over great distances and becomes the most commonly used source of synchronization. The first GPS satellite was launched in 1978 and a full constellation of 24 satellites was achieved in 1994. The satellites have an orbital radius of $16500 \mathrm{~km}$ and go around the earth twice a day. They are arranged in such a way that at least 6 satellites (10 satellites in some locations) are visible at most locations on earth most of the times [4].

The most common use of GPS is to determine the coordinates of the receiver, however, for PMU, one pulseper-second transmitted by the GPS is the most important element. This pulse received by any receiver on earth is coincident with all other pulses received within $1 \mu \mathrm{s}$. These pulses are provided by the accurate clock signals which are maintained by GPS and these clock signals are known as the GPS time which does not take into account the earth's rotation. Corrections to the GPS time are made in the GPS receivers to account for this difference (leap-second correction), so that the receivers provide coordinated universal time. It should be noted that the PMU standard uses UNIX time base with a "second-of-century" (SOC) counter which began counting at midnight on January 1, 1970.

At present there are a number of GPS-like systems being deployed by other nations, with similar goals, mainly including Russia's Global Navigation Satellite System (GLONASS), Europe's GALILEO and China's Beidou Navigation Satellite System. 
Russia's GLONASS also offers a GPS-like feature. Sporadic funding and the resulting incoherent satellite coverage have hampered the widespread acceptance of the GLONASS system, although it is more accurate than GPS in some ways.

Beidou Navigation Satellite System developed by China can both run independently and be compatible with GPS and other satellite navigation systems at the same time [5]. By 2020, it will include 5 geostationary earth orbit (GEO) satellites and 30 non-geostationary earth orbit (Non-GEO) satellites, which could provide services to the entire world. Beidou Navigation Satellite System is referenced to Beidou time, whose bidirectional timing accuracy is $20 \mathrm{~ns}$ and unidirectional timing accuracy is $50 \mathrm{~ns}$, which can fully meet the highest requirement of $1 \mu$ s on the accuracy of time synchronization in power system.

Synchronizing signals can also be disseminated by terrestrial fiber-optic systems. The precision time protocol (PTP) used to synchronize clocks throughout a computer network can achieve clock accuracy in the sub-microsecond range, which is suitable for synchronizing PMUs. PTP version 2 is specified in IEEE Std 1588-2008 [6], and its profile for power system applications is specified in IEEE Std C37.238-2011 [7]. At the top of the time distribution chain, there is a grandmaster clock that synchronizes the clocks in the entire system to coordinated universal time. Each device in the time distribution chain (including Ethernet switches) is required to support IEEE C37.238-2011 to achieve $1 \mu$ s time accuracy. Ethernet switches supporting IEEE C37.238-2011 should perform measurements and corrections for cable delay and residence time.

\section{Standards}

In order to achieve interoperability among PMUs made by different manufacturers, it is essential that all PMUs follow a common standard. A series of IEEE standards [8-14] were issued to ensure the accuracy of synchrophasor measurements and compatibility in data reporting.

The first synchrophasor standard, IEEE Std 1344-1995 [8], was issued in 1995, which included measurement and communication specifications. Based on this standard, PMUs of early manufacturers were tested for interoperability, and it was discovered that their performance at offnominal frequencies was not identical [15], which resulted in the creation of IEEE C37.118-2005 [9]. This standard clarified the requirements for PMU response to off-nominal frequency inputs whose frequency deviation was in the range of $\pm 5 \mathrm{~Hz}$ from the nominal frequency.

In addition, a very important concept of total vector error (TVE) was introduced in IEEE C37.118-2005 to describe the accuracy of synchrophasor measurement [9].
In contrast to magnitude and phase measurements, the errors in a synchrophasor measurement can be due to errors either in the magnitude or in phase, or both. To combine both types of errors into a single measure, TVE is defined as:

$T V E=\sqrt{\frac{\left(\hat{X}_{r}-X_{r}\right)^{2}+\left(\hat{X}_{i}-X_{i}\right)^{2}}{X_{r}^{2}+X_{i}^{2}}}$

where $\hat{X}_{r}$ and $\hat{X}_{i}$ are real and imaginary parts of the estimated (measured) synchrophasor.

It is also important to note that IEEE Std 1344-1995 [8] and IEEE C37.118-2005 [9] did not specify the requirements for response of PMUs to power system transients. Thus, although these two standards played an important role in the steady-state characterization of phasor measurements, they fell short in the aspect of PMU dynamic performance.

Furthermore, in 2009, IEEE approached IEC with a request for dual logo (IEC 61850) for conveying synchrophasor information. This resulted in a joint task force being formed between IEEE and IEC to work on methodologies/agreements that led to changes in IEEE C37.118.2 [11] and the creation of IEC TR 61850-90-5 [16]. The joint IEC/IEEE task force developed a strategy to split IEEE C37.118-2005 into two parts while accommodating a migration to IEC 61850 based technology to meet the North American Synchrophasor Project Initiative (NASPI) requirements.

The events above gave birth to the current version of synchrophasor standards. The standards were created by splitting the previous version, IEEE C37.118-2005, into two parts. The first part, IEEE C37.118.1-2011 [10] focuses on measurements. The second part, IEEE C37.118.2-2011 [11] focuses on the data transfer.

As compared with the previous versions, one major change in IEEE C37.118.1-2011 is the introduction of two performance classes, $\mathrm{P}$ class and $\mathrm{M}$ class, as well as their corresponding dynamic performance requirements. The $\mathrm{P}$ class is mainly for protection and control purposes, which requires fast response, minimum filtering and minimum delay. The $\mathrm{M}$ class is mainly used for measurements in the presence of out-of-band signals, which requires greater precision and significant filtering, and allows slower response and longer delay. Another change is that frequency and ROCOF are introduced as parts of the synchrophasor measurements, and the error limits for frequency and ROCOF in all compliance tests are also defined. In addition, TVE requirements and the compliance tests are expanded by adding new temperature and dynamic performance tests.

In 2014, IEEE C37.118.1a-2014 (amendment 1: modification of selected performance requirements) [14] was 
released to rectify minor inconsistencies of the current synchrophasor standard IEEE C37.118.1-2011 [10].

IEEE C37.118.2-2011 [11] is for addressing real-time communication between PMUs, power dispatching centers (PDCs), and other applications of synchrophasor networks. The main motivation for this standard is the adoption of synchrophasor measurements and integration with other communication protocols. IEEE C37.118.2 adds a new configuration frame to the synchrophasor message format introduced in IEEE C37.118-2005 and defines four message types: data, configuration, header, and command. The standard discusses organization of these four frames, i.e. presenting the structure of each message type, defining the words used in the message, and specifying the number of bytes per word. The standard also defines the time quality codes.

IEEE C37.244-2013 [13] is a guide for functional, performance, and communication needs of PDCs for power system monitoring, protection and control. The guide covers performance and functional requirements of PDCs and defines testing for PDC systems. In the standard, seventeen functional requirements including aspects such as cyber security, protocol support and communication media are described. The PDC performance requirements in terms of latency, robustness, availability and reliability, data processing and environmental compliance are discussed. Besides, the standard outlines various types of tests and test methodologies for evaluation of PDC performance, such as conformance tests and interoperability tests. The standard also includes an informative annex on report rate conversion and filtering issues.

\section{Applications of PMUs and WAMS}

With the emergence and development of synchrophasor technology, the potential applications of PMUs and WAMS in power system monitoring, operation, protection, and control were explored. The applications including model validation, state estimation (SE), protection and close-loop control with synchrophasor measurements are investigated.

\subsection{Model validation and parameters identification}

Many planning and operation tasks such as determination of transmission line operating limits, network expansion, relay settings and the integration of renewable generators are based on the simulation results of power system models. Validation and calibration of power system models and parameters are of great importance due to the fact that these parameters can vary over time either due to intentional adjustments or inadvertent gradual changes. In 2006, the Western Electricity Coordinating Council (WECC) developed a generating unit model validation policy, which required generator models to be validated against recordings every five years [17]. And North American Electric Reliability Council (NERC) required that the models of all generating units be validated every 10 years.

Recent development of synchrophasor technology provides high quality measurements for model validation purposes. In addition to model validation, PMU measurements also allow real-time calibration of current and voltage transformers. Power system models can be validated against recorded dynamics. Discrepancies would reveal inadequacy in the models. The application of PMU measurements for model validation is reported in [18]. It is reported that synchrophasor-based model validation and calibration is a cost-effective way to satisfy the requirements of NERC reliability standards MOD-26, MOD-27, MOD-32, and MOD-33 to verify generator active and reactive power capability and control systems, and to assure their appropriate responses during system disturbances.

In 2006, WECC has adopted provisions to require new generators to install PMUs at the point of interconnection between the power plant and the transmission system, and similar provisions are being considered in other regions to assure that PMU data are collected for model validation.

The construction of WAMS in China also has created the conditions for model calibration and parameter identification. The model and parameter tests of generator excitation system and power system stabilizer (PSS) were carried out in North China, Northeast China and East China power grids. Four artificial short circuit tests have been carried out on the Northeast China power grid on 29 March 2004 and 25 March 2005, which demonstrate the value of synchrophasor measurement based model validation and parameter identification [19].

Up to now, most of the grid operators and transmission owners-including PJM, Peak Reliability Coordinator, California Independent System Operator (CAISO), Bonneville Power Administration (BPA), New York Independent System Operator (ISO), Duke Carolinas, Florida Power and Light, and ERCOT-that received Smart Grid Investment Grants for PMU projects have used their new PMU data to validate and calibrate the system models built inside their state estimators [20]. In addition, Dominion Virginia Power and Virginia Tech have developed a threephase state estimator for calibrating the parameters of Dominion's transmission lines. 


\section{$5.2 \mathrm{SE}$}

The synchrophasor measurement based SE is another area which has gained wide attention of researchers. Synchrophasor technology enables advancements in SE: both algorithmic and architectural. The formulations of several synchrophasor based SE have been described in a recent white-paper [21], e.g. hybrid, linear, distributed and dynamic SE.

The addition of even a few direct measurements of angle to the SE formulation has a number of advantages and creates asymmetry in the problem statement. There are two ways to use PMU data in static SE. The first way, PMU data can be used to augment the conventional supervisory control and data acquisition (SCADA) measurements to build a nonlinear hybrid state estimator handling both the traditional SCADA measurements and the phasor measurements [22]. However, such a SE would still only provide estimated values every 4-5 s.

The second way is using only phasor data in SE. The SE using only phasor data was first proposed in [23, 24]. Because the measurement set consists only of voltage and current synchrophasors and the voltage variables are expressed in the rectangular form, it is a linear SE. The linear SE is also using weighted least squares as the solution algorithm but since the SE formulation is linear then a direct solution can be obtained without the need of iterations, resulting in faster execution of the SE. However, using only phasor data, the observability of the system is difficult to guarantee sometimes.

As the scale of the interconnected power system expands, the problem of "dimensional disaster" in the integral SE is becoming more and more obvious. The distributed SE is performed based on a decentralized architecture and can be realized in a parallel computing way either at an area or at a substation level, reducing dimension and improving the computational efficiency. In distributed SE, the whole system can be divided into multiple subsystems. Each subsystem carries out its own estimation independently and then coordinates the differences between adjacent systems according to the boundary node status to achieve a uniform result. GPS-synchronized measurements make it possible to "distribute" the SE process without the need of additional estimation for coordination because the results of a local state estimator are "globally" valid. Pacific Gas and Electric (PG\&E) have pushed their SE from a central control room out to the substation with the help of PMUs.

Static SE only considers system state on a specific time section, just like taking a snapshot of the system, but dynamic SE based on Kalman filtering techniques is different. It not only considers system's measurement equation, but also establishes the prediction model of the system over time. Therefore, dynamic SE not only provides the system SE, but also forecasts the state of next time step, letting snapshots flowing continuously one by one to track the trajectory of system state over time.

\subsection{Protection systems with phasor inputs}

Synchrophasor measurements have offered solutions to a number of vexing protection problems. The following will introduce the protection systems with phasor inputs, including differential protection, adaptive out-of-step protection, and backup protection, etc.

Differential protection is an effective way to solve the protection problems of the transmission lines equipped with series compensation, flexible alternating current transmission system (FACTS) devices, or multi-terminal lines. However due to the need to ensure the synchronization of input signals at both ends of the protected transmission line, true differential protection was not possible before synchronized phasor measurements. To date, such transmission line problems are solved with 'differential-like' schemes such as phase comparison. The easy availability of synchronized measurements using GPS technology and the improvement in communication technology makes it possible to consider true differential protection of transmission lines and cables, thereby improving line protection performance using phasor measurements from the remote ends of the line.

The existing protection systems are designed to be dependable at the cost of somewhat reduced security. This is a desirable bias when the power system is in a normal state. However, when the system is highly stressed, false trips exacerbate disturbances and lead to cascading events. An attractive solution is to 'adapt' the security-dependability balance in response to changing system conditions as determined by real-time phasor measurements. The concept of 'adaptive relaying' accepts that relays may need to change their characteristics to suit the prevailing power system conditions, which requires the help of PMU to obtain the current state in real time.

Problems with traditional out-of-step relays are found to be unsatisfactory in highly interconnected power networks. This is because the actual electromechanical swings are quite different from the conditions assumed when the relays are set. Wide-area measurements of positive-sequence voltages at networks provide a direct path to determining stability using real-time data instead of using pre-calculated relay settings. Angular swings could be observed directly, and time-series expansions could be used to predict the outcome of an evolving swing. This is the idea of using PMU to realize adaptive out-of-step protection. A related approach was developed for a field trial at the Florida-Georgia interface. 


\subsection{System control with phasor feedback}

Prior to the introduction of real-time phasor measurements, power system control was essentially by local signals. Feedback control with such locally available measurements is widely used in controlling machines. In other situations, control action was taken on the basis of a mathematical model of the system without actual measurement of the system. The advent of phasor measurements allows the consideration of control based on the measured value of remote quantities. It is expected that such control will be less dependent on the model of the system being controlled. The fact that most such phenomena are relatively slow is an encouraging factor for deploying PMUs.

In linear optimal control, the control law will minimize the difference between the output actual trajectory and the desired trajectory. However, the common features of power system control are inherently nonlinear and there are never enough measurements to totally describe the dynamical system. Given phasor measurements of the system, it is possible to measure the difference between the states of system we are actually controlling and the state of a model, thus the predictive control with phasor feedback can be used to solve the nonlinear optimal control problem.

It has been shown that PMU technology has been used for damping low frequency electromechanical oscillations. Generators, high-voltage direct current (HVDC) and FACTS devices can be used as actuators in this regard that work based on modulation-based supplementary control.

In [25] the predictive control was applied to a HVDC system, and the performance was compared with constant current, constant voltage control. Using the same technique, a centralized excitation controller was designed in [26], which requires all the real-time phasor data were brought to a central location. The phasor measurements control the compensating reactance of the transmission line with a thyristor-controlled series capacitor (TCSC) in [27], where a proportional-integral-derivative (PID) controller with an input of the angle difference produced an output signal to control the reactance. In [28] the PSS was used to control inter-area oscillations, and the phasor feedback tolerated in the phasor measurements for PSS was determined to be as much as $150 \mathrm{~ms}$ depending on the frequency of the oscillation.

The existing damping control approaches have been shown to lack robustness. Stabilizers are tuned to damp a specific mode. However once the system changes, the stabilizer is not quite effective. Given the evolving nature of the frequencies and occurrences of the modes, it would be best if some strategy could be devised to provide damping for all modes rather than designing specific controllers aimed precisely at presumed modes. Phasor measurements can provide a solution of 'collocated control' [29] to low-frequency inter-area oscillations in power systems. The term collocated refers to the matrix $\boldsymbol{B}$ appearing with both the vector of control inputs $\boldsymbol{u}$ and the vector of measurements $\boldsymbol{y}$. The compelling reason to use the collocated scheme is its robustness. The collocated form guarantees that the damping added by the feedback does no harm even if the system model changes. In [30] an artificial situation is created to study the effect of collocated control using the HVDC line. The collocated control scheme is to modulate the power flow along the line proportional to the frequency difference between areas measured by the PMUs at either end of the line.

In addition to continuous feedback control, the power system also has another control formation, discrete event control. The control action responds to the state but not continuously. An early phasor measurement application was of this form [31]. The attempt was to control the power flow on the Intermountain and Pacific Intertie HVDC lines in a discrete form in response to a collection of approximately 20 phasor measurements.

The experience of using remote synchrophasor measurements as feedback control signals is not widely reported by the industry. Two main issues in this area are the latency and data drop out. Utilities should invest more attention in solving some of the challenges standing in the way of wide area closed-loop control that include latency issues, data drops, operating system and architectural problems.

\subsection{Remedial action schemes}

As a measure of last resort, system separation into islands is accomplished using "system integrity protection scheme" (SIPS). In many practical situations the prevailing system conditions are quite different from those upon which the SIPS settings are based. Consequently, the performance of these systems may not be optimal. Wide-area real-time synchronized data provide important information on prevailing system conditions to improve the match between SIPS and the actual system state. With the help of real-time data provided by the PMUs, it can be more accurate to determine whether or not a power system is heading to an unstable state, whether or not a network separation is necessary to avoid a blackout, and where the optimal islanding boundaries is. In the absence of prior experience with the prevailing power system state, PMUbased SIPS would lead to islanding operations which are more appropriate for the existing system state.

In SIPS based on wide-area measurement, it is possible to formulate a strategy which would address the issue of load shedding before the frequency begins to decay, or before the voltage begins a dive toward instability. Using 
trained artificial neural networks (ANNs) would produce a decision as to whether or not load shedding is to be invoked. The required wide-area measurements involve tieline flows and phase angles at key network buses. The advantage of load shedding for voltage control would be to consider the voltage problem in its entirety for the power system, and determine appropriate amounts of load to be shed in a coordinated fashion.

\section{PMUs around the world}

In recent years, as the need for the best estimate of the power system's state is recognized to be a crucial element in improving its performance and its resilience in the face of catastrophic failures, phasor measurement technology has gained great attention and rapid development all over the world. The activities related to WAMS and PMU in many countries are summarized briefly below [32].

\subsection{North America}

The first prototype of modern PMU technologies in the world was born at Virginia Tech in early 1980s. The 2003 blackout in the Northeast of the United States and Canada has been a major driver in taking steps to improve reliability of the North American grid with the help of synchronized measurement. Because the PMU data captured during the blackout were used for the event analysis, the U.S. Department of Energy (DOE), partnered with eastern utilities, launched the Eastern Interconnection Phasor Project (EIPP) to deploy a wide-area phasor network in the eastern power grid. EIPP started by sending data from several PMU systems to the Tennessee Valley Authority's (TVA) central PDC, and many companies have been involved with EIPP, such as Ameren, AEP, American Transmission Company, etc.

In the western part of the U.S., the research and prototype testing efforts by the California ISO began in 2002. In parallel, the deployment of real-time PMU data analysis, dynamic stability assessment and data visualization applications was further enhanced by adopting the latest technology at BPA, PG\&E, Southern California Edison (SCE), and Western Area Power Administration (WAPA). Currently, so many other companies have been involved, such as Sempra Utilities, ESBI Alberta, Salt River Project (SRP), etc. Since early 2007, the two efforts from eastern and western North America have been combined to become the North American Synchrophasor Initiative (NASPI) that also covers Canada and Mexico.

With the participation and support of transmission operator (TO), there have been many synchrophasors projects organized by ISO or regional utility. There have be
420 PMUs installed in North-East, 400 PMUs in Midwest, 150 PMUs in South, 120 PMUs in Texas, 500 PMUs in WECC and at least 300 PMUs in Mexico. Figure 4 shows the current development of PMU in North America. As shown in Fig. 4, there are many controlling organizations such as Electric Reliability Council of Texas (ERCOT), Southern Company (SOCO), TVA, etc. and the areas for the controlling organizations are indicated by the light blue ovals. The other little squares and spots are the locations of measuring devices and data collection.

The experiences from deployed PMU systems have already proven that the synchronized measurement technology is required to accurately analyze and control the North American power grid performance both in real time and offline. PMU technology has been beneficial for postdisturbance data analysis and early warning systems, improving system models for faster system restoration, etc.

\subsection{Mexico}

PMUs have been in use in Mexico since the 1990s. Initial interest was in observing the power system through the eyes of the high-precision PMUs. The data obtained from PMUs over years have been invaluable in observing underdamped low-frequency oscillations on the network and the performance of protection systems during power system disturbances.

On 10 March 2005 two of the four electric systems in Mexico (Northwest Grid and the National Grid), which had operated asynchronously until that date were synchronized. As soon as the tie lines were connected, sustained interarea oscillations were observed throughout the system, which would eventually lead to the separation of the system again. Thanks to WAMS, it was possible to record and simulate the oscillations that were present during the synchronous operation. PMUs also make possible direct visualization of power system frequency, voltage, phase angle, active and reactive power, etc. However, to find the

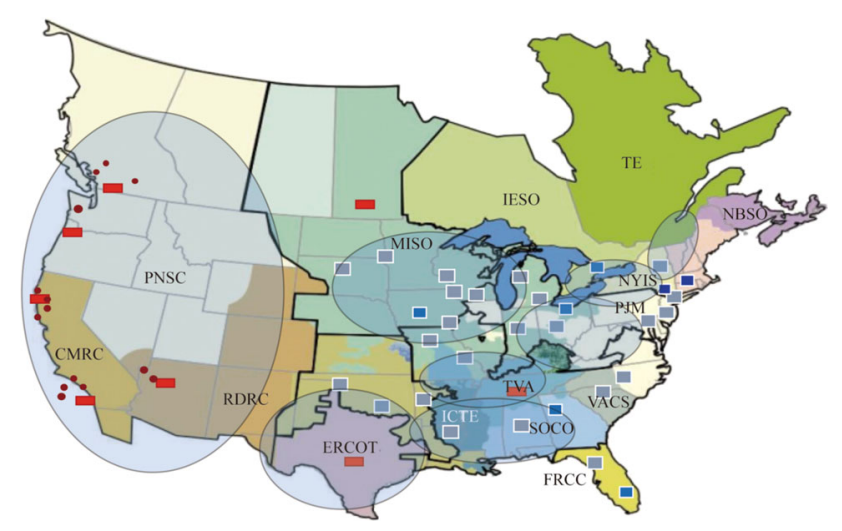

Fig. 4 Current development of PMU in North America 
origin of the oscillations and to correct the settings for excitation controls and PSSs of generators, it was necessary to calculate the curves of real power versus frequency $(P$ $F)$ and reactive power versus voltage $(Q-V)$ of machines and connection circuits among systems.

In the future, using PMUs, some offline analysis will be implemented in real time to detect risks of instability, such as establishing limits with alarms for oscillations and calculating the curves of $P-F$ and $Q-V$ for generators during various levels of load, generation, and system configurations. Also, in the future it will be possible to use the threedimensional analysis of oscillations in transmission lines and the dynamic behavior of distance relays.

\subsection{Brazil}

Since late 2000, the Brazilian ISO ONS has launched two WAMS-related projects aiming to implement a largescale synchronized phasor measurement system (SPMS) for both offline and real-time applications: a phasor recording system to record system dynamics during long duration wide-area disturbances, and an application of phasor measurement data for real-time system operation decision making to extend the initial SPMS for control center real-time applications.

The project has been progressing well and has achieved many objectives, such as system design and specifications and proof-of-concept pilot application implementations. The system architecture of the SPMS includes PMU, main ONS PDC and state power dispatching center (SPDC) in each substation. Some related qualifying tests are also deployed to verify the correct and consistent performance of all PMUs deployed by the different utilities and the compliance of the SPDC and main PDCs. And four candidate applications of phasor measurement technology were chosen for a proof-of-concept pilot implementation, of which main goal is to monitor system oscillations with poor damping, to monitor the stresses of the electric power transmission system, to resynchronize islands and to close loops in parts of the Brazilian National Interconnect Power System (SIN), respectively.

\subsection{Europe}

In the past few years, PMUs and WAMSs in Europe had extensive development, nearly installed in every country. In Italy, planning was on the way to install 30 PMUs. In Sweden, an experimental network of three PMUs was installed in three universities. Denmark had a two PMU setup for research purposes. Swiss also deployed their WAMS. The common characteristics of these devices are following: high time resolution (20-100 ms), high accuracy of voltage and current (class 0.2 ) and precise time synchronization (GPS or DCF77).

The PMUs in Europe are distributed in seven regions of the continent. Through the defense plan, Europe has improved control with synchronized phasors. Real time phase angle differences of a region with the rest of the country are monitored at the center. The tie lines of the region will break off from the rest of the country once angle separation exceeds limits.

Inside the UCTE, the WAMS measurements are exchanged between the transmission system operators (TSOs) for calibration of system dynamic models or postmortem analysis. One major focus currently is the permanent monitoring of the sporadic appearance of poorly damped inter-area oscillations. Activities of exchanging online measurements between several data concentrators have already started and are in the phase of further extensions. Current partners of these plans are the TSOs from Austria, Croatia, Greece, Italy, Slovenia, and Switzerland. The additional basic functions can be summarized as: voltage phase angle difference monitoring, line thermal monitoring, voltage stability monitoring, and online monitoring of system damping, etc.

\subsection{China}

The installation of PMUs in the Chinese power grid began in 1995. From 1995 to 2002, about 30-40 PMUs were installed and the main data concentrator stations of WAMS were established in East China, South China, Northwest China, Sichuan power grids, and the SPDC successively. The installed PMU successfully recorded dynamic processes of low-frequency oscillations in the Chinese power grid. By the end of 2002, Chinese manufacturers began to offer commercial PMUs.

$\mathrm{Up}$ to now, all $500 \mathrm{kV}$ and above substations, some important $220 \mathrm{kV}$ substations and $100 \mathrm{MW}$ and above power plants in the Chinese power grid have installed PMUs. In total, over 3000 PMUs have been commissioned in the practical system. And WAMSs have been established in all provincial grids except for Tibet.

In China, the main station of the WAMS is located at the regional or provincial dispatching center and composed of an advanced application station, database server, and data concentrator. The advanced application station retrieves data from a data concentrator via local area network (LAN) instead of Ethernet, which reduces the time delay of the data. Currently, some data concentrators have already contained 5000 phasor measurements with the $100 \mathrm{~Hz}$ storage rate.

In China, synchrophasor measurements based low-frequency oscillation damping control system has passed the field test. Other PMU applications, such as state estimator, 
security assessment, adaptive protection, and emergency control are also currently undergoing development.

\subsection{India}

The Indian power system is growing at an accelerated pace. The power system is divided into five electrical regions: Northern, Eastern, Western, Northeastern and Southern. So far they have been well synchronized, especially Southern is connected with the rest by back-to-back HVDC. In the past few years, various large-capacity power plants of about $4000 \mathrm{MW}$ each have been set up, and a hybrid system comprising $6800 \mathrm{kV}, 6000 \mathrm{MW}$ HVDC and $765 \mathrm{kV}$ AC system has been developed. The peak demand would be further expected to increase to over $500 \mathrm{GW}$ by 2027 , for which total installed capacity of about $700 \mathrm{GW}$ would be required.

With the rapid growth of the power system, it is important to know the dynamic state of the grid, which calls for the development of an intelligent grid comprising a WAMS, adaptive islanding, and self-healing aspects. To achieve this objective, POWERGRID, the central transmission utility, initiated the work for development of an intelligent grid comprising a WAMS, Remedial Action Scheme (RAS), SIPS, etc. In the first stage, a few PMUs (four to five in each region) were installed at critical buses in all the regional grids. In the second stage, about 1500 PMUs are being installed at various buses, and in the final step, up to 3000 additional PMUs will be installed at all EHV and HVDC substations. SE, various calibration and parameter estimation functions are to be installed, and a SIPS for regulation and control purposes is to be developed.

\subsection{Russia}

Synchronous interconnection of the 14 national power systems of Eastern Europe, Central Asia, and Siberia from the western borders of the Ukraine to Baikal and from Tajikistan to Kola Peninsula has been achieved. It is the most geographically extended power system in the world, spanning eight time zones.

Dynamic behavior investigation of such an extended power system needs information on electromechanical transient parameters with resolution of $0.02-0.2 \mathrm{~s}$ and synchronized by space satellite time tags. Such information is provided by interconnected power system/unified power system (IPS/UPS) WAMSs. Development of this system started in 2005. Currently 45 PMUs are located in the major power plants and substations from the east to the west and from the south to the north of this immense interconnected power grid. Three models of PMUs are being used in this grid: Smart WAMS (RTSoft, Russia),
Regina (ANIGER, Ukraine) devices, and Powerlog (AENEA, Germany).

The Russian WAMS has the following three-tier control structure: the first level contains the multifunctional measuring transducers and communication server installed at the substations; the second level is the control centers allocated in the interconnected dispatch offices (IDOs) of the UPS of Russia and dispatch centers of the IPS; and the highest level is the system operator central dispatch office of UPS.

As an effective tool of the dynamic performance investigation of synchronous interconnection, phasor measurements in the IPS/UPS are currently used primarily for system performance monitoring and analysis, including the reference dynamic model (RDM) validation, low-frequency oscillation monitoring and power system control in real time, allowing system operators to create new schemes and algorithms for power system emergency control.

Open Access This article is distributed under the terms of the Creative Commons Attribution 4.0 International License (http:// creativecommons.org/licenses/by/4.0/), which permits unrestricted use, distribution, and reproduction in any medium, provided you give appropriate credit to the original author(s) and the source, provide a link to the Creative Commons license, and indicate if changes were made.

\section{Appendix A}

Network simulator at AEP for development of computer based distance relay is shown in Fig. A1.

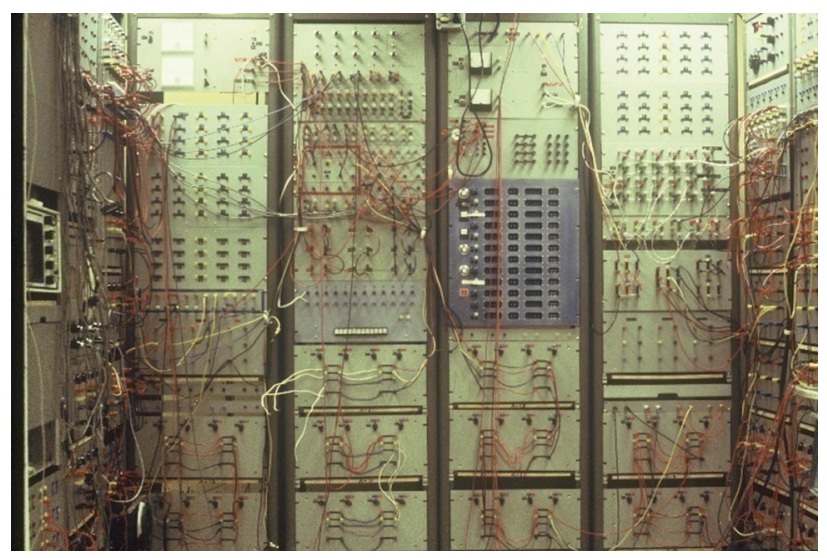

Fig. A1 Network simulator at AEP for development of computer based distance relay

\section{References}

[1] Missout G, Girard P (1980) Measurement of bus voltage angle between Montreal and SEPT-ILES. IEEE Trans Power App Syst PAS-99(2):536-539 
[2] Missout G, Beland J, Bedard G (1981) Dynamic measurement of the absolute voltage angle on long transmission lines. IEEE Trans Power App Syst PAS-100(11):4428-4434

[3] Bonanomi P (1981) Phase angle measurements with synchronized clocks-principles and applications. IEEE Trans Power App Syst PAS-100(12):5036-5043

[4] Phadke AG, Thorp JS (2008) Synchronized phasor measurements and their applications, 2nd edn. Springer, New York

[5] China Satellite Navigation System Management Office (2013) Development report for Beidou Satellite Navigation System (version 2.2). Beijing

[6] IEEE Standard 1588: IEEE standard for a precision clock synchronization protocol for networked measurement and control systems (revision of IEEE Std 1588-2002), 2008

[7] IEEE Std C37.238: IEEE standard profile for use of IEEE 1588 precision time protocol in power system applications, 2011

[8] IEEE Standard 1344: IEEE standard for synchrophasors for power systems, 1995

[9] IEEE Standard C37.118: IEEE standard for synchrophasors for power systems, 2005

[10] IEEE Standard C37.118.1: IEEE standard for synchrophasor measurements for power systems, 2011

[11] IEEE Standard C37.118.2: IEEE standard for synchrophasor data transfer for power systems, 2011

[12] IEEE Standard C37.242: IEEE guide for synchronization, calibration, testing, and installation of phasor measurement units (PMUs) for power system protection and control, 2013

[13] IEEE Standard C37.244: IEEE guide for phasor data concentrator requirements for power system protection, control, and monitoring, 2013

[14] IEEE Std C37.118.1a: IEEE standard for synchrophasor measurements for power systems (amendment 1: modification of selected performance requirements), 2014

[15] Depablos J, Centeno V, Phadke AG et al (2004) Comparative testing of synchronized phasor measurement units. In: Proceedings of IEEE power engineering society general meeting, Denver, USA, 6-10 June 2004, pp 948-954

[16] IEC Standard TR 61850-90-5: use of IEC 61850 to transmit synchrophasor information according to IEEE C37.118, 2012

[17] Western Electricity Coordinating Council (2005) WECC generating unit model validation policy. http://www.wecc.biz/ documents/2005/Standards/Generator_Model_Validation_. Policy_Statement_01282005.pdf. Accessed 28 January 2005

[18] North American synchrophasor initiative, model validation using phasor measurement unit data. NSPI Technical Report: TR-2015-004, 1 March 2015

[19] Zhang DX, Tang Y, Zhu F et al (2007) Study on load model validation and its adjustment approach based on calibration of simulations against disturbances. Power Syst Technol 31(4):24-31

[20] Annakage UD, Mehrizi-Sani A, Rajapakse A et al (2017) Application of phasor measurement unit for monitoring power system dynamic performance. Paris

[21] Farantatos E. State estimation advancements enabled by synchrophasor technology. EPRI Whitepaper
[22] Gol M, Abur A (2015) A hybrid state estimator for systems with limited number of PMUs. IEEE Trans Power Syst 30(3):1511-1517

[23] Thorp JS, Phadke AG, Karimi KJ (1985) Real time voltagephasor measurements for static state estimation. IEEE Trans Power App Syst PAS-104(11):3098-3106

[24] Phadke AG, Thorp JS, Karimi KJ (1986) State estimation with phasor measurements. IEEE Power Eng Rev PER 6(2):233-241

[25] Rostamkolai N, Phadke AG, Thorp JS et al (1988) Measurement based optimal control of high voltage AC/DC systems. IEEE Trans Power Syst 3(3):1139-1145

[26] Manansala EC, Phadke AG (1991) An optimal centralized controller with nonlinear voltage control. Electr Mach Power Syst 19(2):139-156

[27] Smith MA (1994) Improved dynamic stability using FACTS devices with phasor measurement feedback. Dissertation, Virginia Tech

[28] Mili L, Baldwin T, Phadke AG (1991) Phasor measurements for voltage and transient stability monitoring and control. In: Proceedings of workshop on application of advanced mathematics to power systems, San Francisco, USA, 4-6 September 1991

[29] Liu J, Thorp JS, Chiang HD (1992) Modal control of large flexible space structures using collocated actuators and sensors. IEEE Trans Autom Control 37(1):143-147

[30] Rovnyak S, Taylor CW, Mechenbier JR et al (2007) Plans to demonstrate decision tree control using phasor measurements for HVDC. http://phasors.pnl.gov/Meetings/2007_may/presentations/ synch_freq_meas.pdf. Accessed 7 May 2007

[31] Rovnyak S, Taylor CW, Mechenbier JR et al (1995) Plans to demonstrate decision tree control using phasor measurements for HVDC fast power changes. In: Proceedings of conference on fault and disturbance analysis and precise measurements in power systems, Arlington, USA, 9 November 1995

[32] Phadke AG (2008) The wide world of wide-area measurement. IEEE Power Energ Mag 6(5):52-65

Arun G. PHADKE is a University Distinguished Research Professor at Virginia Tech in Blacksburg, Virginia, USA. He is a Fellow of IEEE. He was elected to the U.S. National Academy of Engineering in 1993. He was awarded Honorary Doctorate by INP Grenoble, France in 2006. He received the Benjamin Franklin Medal in 2008, and the IEEE Power Engineering Medal in 2016. His primary research area is the microcomputer based monitoring, protection, and control of power systems.

Tianshu BI received her Ph.D. degree at the Department of EEE in the University of Hong Kong in 2002. She is currently a professor at North China Electric Power University. She got the honor of The National Science Fund for Distinguished Young Scholars in 2017 and National High-level Personnel of Special Support Program in 2016. Her research interests include power system protection and control, synchronized phasor measurement technique and its application. 